DrAft VERSion April 22, 2014

Preprint typeset using $\mathrm{LAT}_{\mathrm{E}} \mathrm{X}$ style emulateapj v. 5/2/11

\title{
STRONG DEPENDENCE OF THE INNER EDGE OF THE HABITABLE ZONE ON PLANETARY ROTATION RATE
}

\author{
JUN YANG \\ Department of Geophysical Sciences, University of Chicago, Chicago, IL, USA
}

GWenä̈L Boué

Department of Astronomy \& Astrophysics, University of Chicago, Chicago, IL, USA and Sorbonne Universités, UPMC Univ Paris 06, UMR 8028, IMCCE, Observatoire de Paris, F-75014 Paris, France

\author{
DANIEL C. FABRYCKY \\ Department of Astronomy \& Astrophysics, University of Chicago, Chicago, IL, USA \\ AND \\ DORIAN S. ABBot \\ Department of the Geophysical Sciences, University of Chicago, Chicago, IL, USA \\ Draft version April 22, 2014
}

\begin{abstract}
Planetary rotation rate is a key parameter in determining atmospheric circulation and hence the spatial pattern of clouds. Since clouds can exert a dominant control on planetary radiation balance, rotation rate could be critical for determining mean planetary climate. Here we investigate this idea using a three-dimensional general circulation model with a sophisticated cloud scheme. We find that slowly rotating planets (like Venus) can maintain an Earth-like climate at nearly twice the stellar flux as rapidly rotating planets (like Earth). This suggests that many exoplanets previously believed to be too hot may actually be habitable, depending on their rotation rate. The explanation for this behavior is that slowly rotating planets have a weak Coriolis force and long daytime illumination, which promotes strong convergence and convection in the substellar region. This produces a large area of optically thick clouds, which greatly increases the planetary albedo. In contrast, on rapidly rotating planets a much narrower belt of clouds form in the deep tropics, leading to a relatively low albedo. A particularly striking example of the importance of rotation rate suggested by our simulations is that a planet with modern Earth's atmosphere, in Venus' orbit, and with modern Venus' (slow) rotation rate would be habitable. This would imply that if Venus went through a runaway greenhouse, it had a higher rotation rate at that time.
\end{abstract}

Subject headings: astrobiology - planets and satellites: atmospheres - planets and satellites: detection

\section{INTRODUCTION}

It is traditional to define the habitable zone based on whether liquid water can be maintained on a planet's surface, which is primarily controlled by the planet's surface temperature (Kasting et al. 1993, 2014). Accurate estimates of the stellar flux boundaries of the habitable zone are critical for estimating parameters of astrophysical interest such as the frequency of Earth-like planets (e.g., Kopparapu 2013). The inner edge of the habitable zone is set by the runaway greenhouse effect, a positive feedback through which an entire ocean can be evaporated into the atmosphere (Nakajima et al. 1992). Our ability to constrain the stellar flux corresponding to the inner edge of the habitable zone has been severely hampered by the inability of $1 \mathrm{D}$ radiative-convective models to predict cloud behavior (Selsis et al. 2007).

Clouds are critical to planetary energy balance. Cloud reflection of solar radiation is responsible for most of the planetary albedo on modern Earth (Donohoe \& Battisti 2011), and clouds also significantly increase Earth's greenhouse effect by absorbing terrestrial infrared emis-

Correspondence: abbot@uchicago.edu sion (Harrison et al. 1990). Cloud coverage and location are primarily controlled by large-scale atmospheric circulation, which is determined by a variety of factors including stellar flux, orbital parameters, and rotation rate. As the stellar flux increases, cloud coverage and thickness may increase, potentially leading to a higher albedo and a negative feedback, or decrease, potentially leading to a lower albedo and a positive feedback 1 . Orbital parameters such as obliquity and eccentricity can both drive large-amplitude seasonal cycles of the atmospheric circulation and surface temperature (e.g., Ferreira et al. 2014), but they tend to minimally affect the annual-mean climate (Williams \& Pollard 2002, 2003).

Planetary rotation rate determines the strength of the Coriolis force (the apparent force felt due to the rotation of the planet) and the length of day (and night). The Coriolis force is a key parameter in determining the atmospheric circulation (e.g., Schneider 2006; Showman et al. 2013). If the Coriolis force is strong, thermally direct

${ }^{1}$ Here we assume that changes in cloud reflection of stellar radiation (cooling) dominate over changes in cloud absorption of planetary infrared radiation (warming), which is the case in the simulations we present below. 

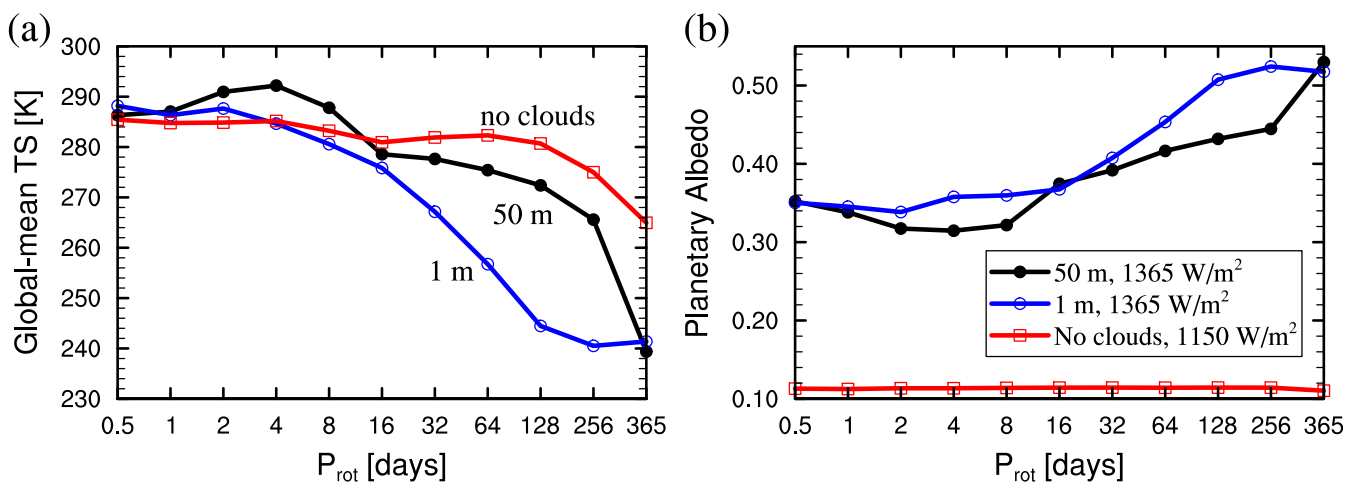

(c)

(d)
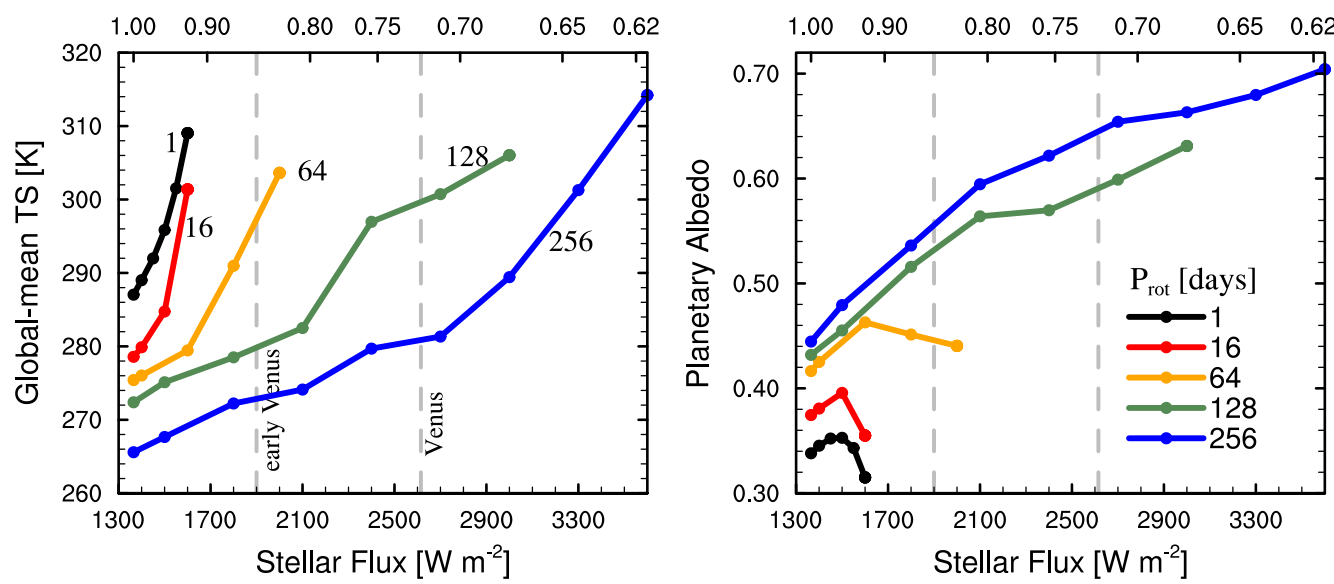

FIG. 1. - This figure demonstrates the dependence of planetary climate on rotation period $\left(P_{\text {rot }}\right)$ for planets orbiting a Sun-like star. (a) and (b): Global-mean surface temperature (TS) and planetary albedo as a function of $P_{\text {rot }}$ for a given stellar flux $\left(S_{0}\right)$. Black line: $S_{0}=1365 \mathrm{~W} \mathrm{~m}^{-2}$ and the surface heat capacity (D) is equivalent to $50 \mathrm{~m}$ of water; blue line: $S_{0}=1365 \mathrm{~W} \mathrm{~m}{ }^{-2}$ and $\mathrm{D}=1 \mathrm{~m}$; red line: clouds are switched off, $S_{0}=1150 \mathrm{~W} \mathrm{~m}^{-2}$, and D $=50 \mathrm{~m}$. For $P_{\text {rot }}=365$ days, the planet is in a synchronously rotating state. (c) and (d): Global-mean surface temperature (TS) and planetary albedo as a function of stellar flux $\left(S_{0}\right)$ for a given $P_{\text {rot }}$ with $\mathrm{D}=50 \mathrm{~m}$. The vertical dashed lines denote the stellar flux of early and modern Venus. The upper horizontal axis in (c-d) is the corresponding semi-major axis between a Sun-like star and the planet in AU. In all these simulations the orbital period is 365 days and there is no sea ice.

latitudinal circulations (Hadley cells) are constrained to low latitudes, and the atmosphere organizes into banded, roughly longitudinally symmetric regions. If the Coriolis force is weak, horizontal temperature gradients become small throughout the atmosphere and the Hadley cells can extend globally. The length of day, combined with surface and atmospheric thermal inertia, helps determine the surface temperature distribution, which drives atmospheric circulation (Pierrehumbert 2010). For a short day (or large thermal inertia), the surface temperature difference between day and night is small. If the day is long enough that the dayside is much warmer than the nightside, atmospheric circulation is characterized by ascent on the warm dayside and descent on the cold nightside.

Recently a number of calculations have been done with 3D general circulation models (GCMs) to assess the effects of atmospheric circulation, subsaturation, and clouds on the inner edge of the habitable zone Yang et al. 2013; Leconte et al. 2013b; Wolf \& Toon 2014). In the extreme case of tidally locked synchronously rotating planets orbiting M-stars, strong atmospheric ascent on the dayside leads to thick dayside cloud coverage and a very high planetary albedo (Yang et al. 2013). This can allow a planet to remain habitable at twice the stellar flux 1D model calculations would suggest. In contrast, for rapidly rotating planets with banded atmospheric circulations, cloud behavior remains roughly similar to modern Earth's (which the albedo of $1 \mathrm{D}$ models are tuned to) so that the inner edge of the habitable zone in 3D models is similar to that in 1D models (Leconte et al. 2013b; Wolf \& Toon 2014). Another interesting difference is that the cloud feedback near the runaway greenhouse threshold appears to be negative for tidally locked planets (Yang et al. 2013) and positive for rapidly rotating planets (Leconte et al. 2013b; Wolf \& Toon 2014)2.

The purpose of this study is to investigate the effects of a range of planetary rotation rates, between tidally locked and rapidly rotating, and stellar types on cloud behavior and the inner edge of the habitable zone. To do this we use a 3D GCM with a sophisticated cloud scheme that reproduces cloud behavior well in the large range of climates observed on modern Earth. Although we do not push the model significantly outside of this range, it is important to note that cloud modeling is difficult, and other models may yield quantitatively different results. Nevertheless, we focus on results due to robust physi-

${ }^{2}$ Wolf \& Toon (2014) find a cloud feedback that starts negative, then becomes positive near the runaway greenhouse. 


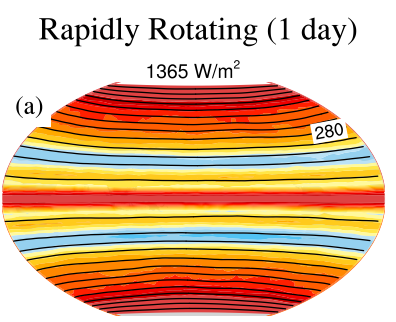

$1600 \mathrm{~W} / \mathrm{m}^{2}$

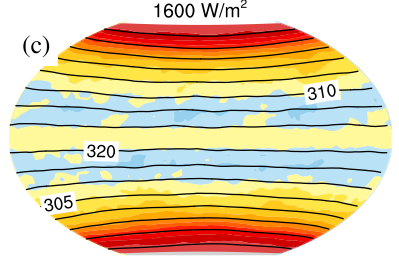

$1365 \mathrm{~W} / \mathrm{m}^{2}$

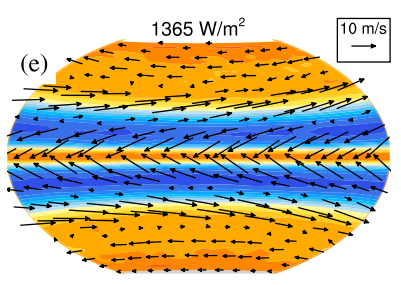

$1600 \mathrm{~W} / \mathrm{m}^{2}$

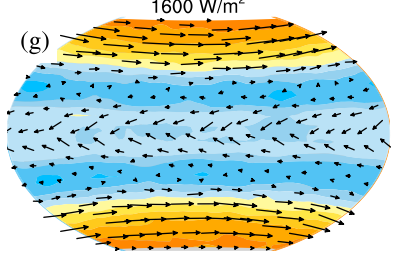

Slowly Rotating (128 days)
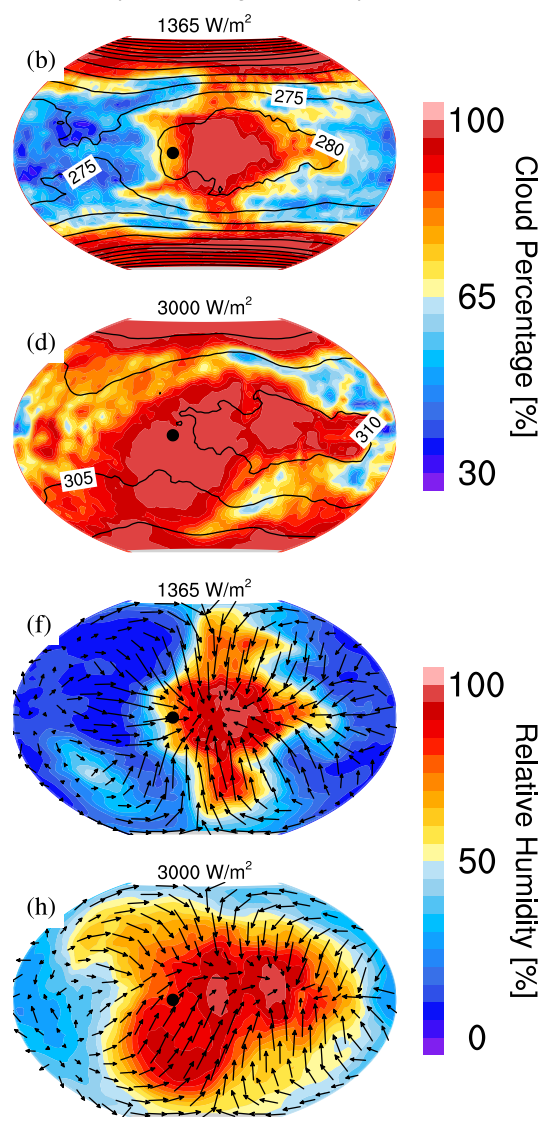

FIG. 2.- Differences in clouds and atmospheric circulation between rapidly (left) and slowly (right) rotating planets with an orbital period of 365 days. The rotation period is 1 day for the rapidly rotating planet and the stellar flux is 1365 or $1600 \mathrm{~W} \mathrm{~m}^{-2}$. The rotation period is 128 days for the slowly rotating planet and the stellar flux is 1365 or $3000 \mathrm{~W} \mathrm{~m}^{-2}$. (a-d): Total cloud coverage (\%, shaded) and surface temperature (K, black contours with an interval of $5 \mathrm{~K}$ ). (e- $\mathrm{h})$ : Relative humidity at 450 mbar (\%, shaded) and near-surface winds ( $\mathrm{m} \mathrm{s}^{-1}$, vectors). The black dot in the right panels is the transient substellar point, which moves westward around the planet with a period of 197 days. All variables are averaged over 30 days.

cal processes that should be qualitatively similar in any $3 \mathrm{D}$ model. Our main conclusion is that for all stellar types slowly rotating planets (orbital period $\approx 100$ days or more) behave similarly to tidally locked planets and have a high planetary albedo near the inner edge of the habitable zone. The width of the habitable zone is therefore strongly dependent on planetary rotation rate.

\section{METHODS}

For most of the results presented below we use the Community Atmosphere Model version 3.1 (CAM3, Collins et al. 2004), which is a 3D atmospheric general circulation model (GCM) that was developed by the National Center for Atmospheric Research to simulate the climate of Earth. CAM3 calculates atmospheric circulation and radiative transfer, and uses subgrid-scale parameterizations to model small-scale vertical convection, clouds, and precipitation. We perform additional simulations in some cases using the Community Atmosphere Model version 4.0 (CAM4, Neale et al. 2010) and the Community Climate System Model version 3.0 (CCSM3, Collins et al. 2006). CCSM3 is a coupled ocean-atmosphere model that calculates ocean circulation explicitly and uses CAM3 as its atmospheric component. We run CAM3 and CAM4 coupled to an immobile ocean with a uniform depth and a uniform albedo of 0.06. The GCMs simulate marine stratus, layered, shallow convective, and deep convective clouds as well as prognostically calculate liquid and ice cloud condensate. Compared to CAM3, CAM4 has a revised deep convection scheme and a similar cloud scheme. The default atmospheric pressure we use is 1.0 bar (mainly $\mathrm{N}_{2}$ ), with $\mathrm{CO}_{2}=400$ ppmv and $\mathrm{CH}_{4}=1$ ppmv. We set geothermal heat flux, aerosols, obliquity, and eccentricity to zero. We run CAM3 at a horizontal resolution of $3.75^{\circ} \times 3.75^{\circ}$ with 26 vertical levels from the surface to $\approx 30 \mathrm{~km}$.

In our simulations where we change the rotation rate and increase the stellar flux we use Earth's planetary parameters, including radius $\left(R_{\oplus}\right)$, gravity $\left(g_{\oplus}\right)$, and orbital period ( $P_{\text {orb }}, 365$ days). We increase the stellar flux until the model crashes, which occurs when the global mean surface temperature reaches $\approx 310 \mathrm{~K}$. Comparison with the clear-sky calculations of Leconte et al. (2013b) shows agreement between CAM3 and generic-LMD to within $\approx 5 \mathrm{~K}$ up to this temperature. We use the last converged solution as a proxy for the inner edge of the habitable zone. We examine a series of rotation periods $\left(P_{\text {rot }}\right)$ ranging from 12 hours to 365 days. We perform simulations using the Sun's spectrum and stellar spectra corresponding to M (blackbody $3400 \mathrm{~K}), \mathrm{K}(4500 \mathrm{~K})$, and $\mathrm{F}(6800 \mathrm{~K})$ stars. In our simulations examining a planet with Venus' orbital characteristics and Earth's atmosphere, we use a stellar flux $S_{0}=2615 \mathrm{~W} \mathrm{~m}^{-2}$, $P_{\text {orb }}=225$ days, $P_{\text {rot }}=-243$ days (retrograde rotation), 


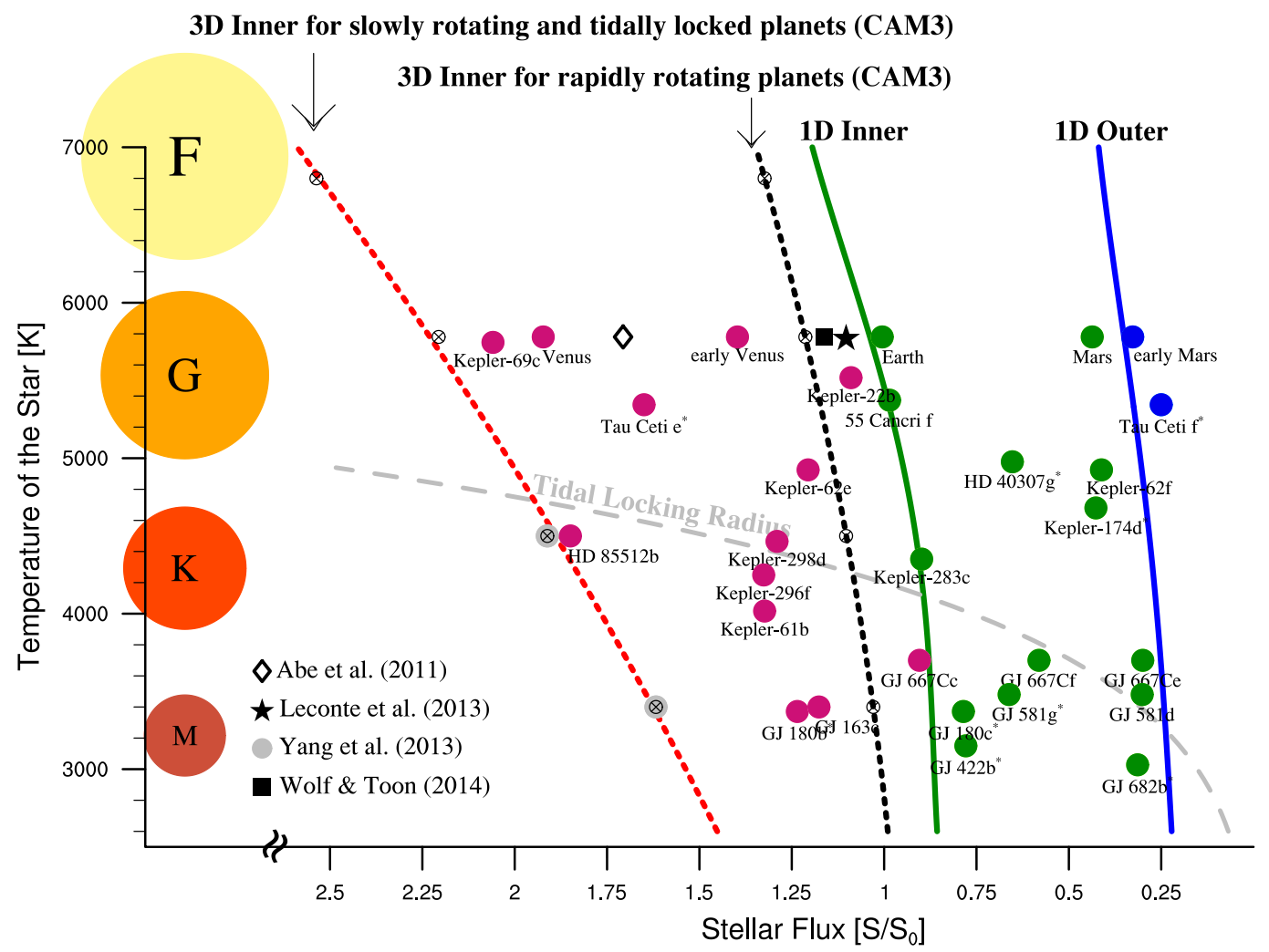

FIG. 3.- Habitable zone boundaries as a function of stellar type and planetary rotation rate for a 1D radiative-convective model and for the 3D general circulation model CAM3. Blue line: the 1D outer edge (maximum greenhouse, Kopparapu et al. 2013); green line: the 1D inner edge (runaway greenhouse, Kopparapu et al. 2013); black line: the 3D inner edge for rapidly rotating planets in CAM3 (rotation period of 1 day); red line: the 3D inner edge for slowly rotating planets in CAM3 (rotation period of 128 days for G and F stars, and tidally locked with an orbit of 60 days for $\mathrm{M}$ and $\mathrm{K}$ stars); gray line: the tidal locking radius (Kasting et al. 1993). The CAM3 simulations used to calculate the 3D inner edge lines are denoted by $\otimes$. We also plot the inner edge of the habitable zone for rapidly rotating dry planets (Abe et al. 2011), for Earth obtained in generic-LMD (Leconte et al.,2013b) and CAM3 with a modified radiative-transfer module (Wolf \& Toon 2014). Finally, we plot solar system planets and discovered exoplanets (unconfirmed exoplanets are marked by *).

$R_{p}=0.95 R_{\oplus}$, and $g_{p}=0.9 g_{\oplus}$. Our default simulation in this case uses Earth's continental configuration with continents composed of clay and sand $($ albedo $=0.2$, thermal conductivity $=0.26 \mathrm{~W} \mathrm{~m}^{-1} \mathrm{~K}^{-1}$ ).

\section{DRAMATIC EFFECT OF PLANETARY ROTATION}

For a given stellar flux $\left(S_{0}\right)$, the surface temperature (TS) of rapidly rotating planets is much higher than that of slowly rotating planets (Fig. 1a). When we use a surface heat capacity equivalent to $50 \mathrm{~m}$ of water $(\mathrm{D}=50 \mathrm{~m})$, the global-mean TS decreases by $20 \mathrm{~K}$ when we increase the rotation period $\left(P_{\text {rot }}\right)$ from 1 day to 256 days, and then decreases by another $25 \mathrm{~K}$ when the planet becomes tidally locked $\left(P_{r o t}=365\right.$ days $)$. The primary reason for this is that the cloud albedo is much higher on slowly rotating planets (Fig. 1b). If we artificially set clouds to zero in the model, TS is nearly independent of rotation rate, except for the tidally locked and nearly tidally locked cases (Fig. 1a). TS is lower in these cases because a thermal inversion on the nighside leads to a radiator fin that cools the planet (Yang et al. 2013; Yang \& Abbot 2014). If we reduce $\mathrm{D}$ to $1 \mathrm{~m}$, the TS distribution is able to adjust to the moving stellar forcing so that the planet can behave as if it were tidally locked even when $P_{\text {rot }}$ is decreased to 128 days (Fig. 1 ). When $\mathrm{D}=50 \mathrm{~m}$, TS has a maximum at $P_{\text {rot }}=4$ days that appears to be associated with a high latitude cloud feedback similar to that described by Abbot \& Tziperman (2008).

There is a dramatic difference between the response of the planetary albedo to increases in $S_{0}$ for rapidly and slowly rotating planets. For rapidly rotating planets, as $S_{0}$ increases the planetary albedo first increases, then decreases (Fig. 1d), leading to a positive feedback near the runaway greenhouse threshold (Leconte et al. 2013b; Wolf \& Toon 2014). This positive feedback is due to a combination of decreased cloud reflection and increased water vapor absorption. In contrast, for slowly rotating planets, the planetary albedo monotonously increases with $S_{0}$, leading to a negative feedback that stabilizes the climate. For rapidly rotating planets, the atmospheric circulation is banded and Earth-like. This leads to high cloud coverage both in a tropical convergence zone associated with the ascent of the Hadley cells (Fig. 2e) and at higher latitudes associated with baroclinic eddies (Fig. 2a). The tropical clouds are most important for planetary albedo because the stellar flux is highest there. As $S_{0}$ increases, the equator-to-polar temperature gradient decreases (Fig. 2c), which weakens the Hadley cells (Held \& Hou 1980), reduces tropical cloud coverage (Fig. 2c), and decreases the planetary albedo (Fig. 1d). For slowly rotating planets, a global atmospheric circulation occurs with strong low-level convergence and ascent in the (slowly moving) substellar region (Fig. 2f) similar to the circulation on synchronously rotating planets (Joshi et al. 1997; Showman et al. 2013; Leconte et al. 2013a). This circulation leads to strong convection and optically thick clouds (Fig. 2b) in the substellar region (Yang et al. 2013). As $S_{0}$ increases the circulation weak- 

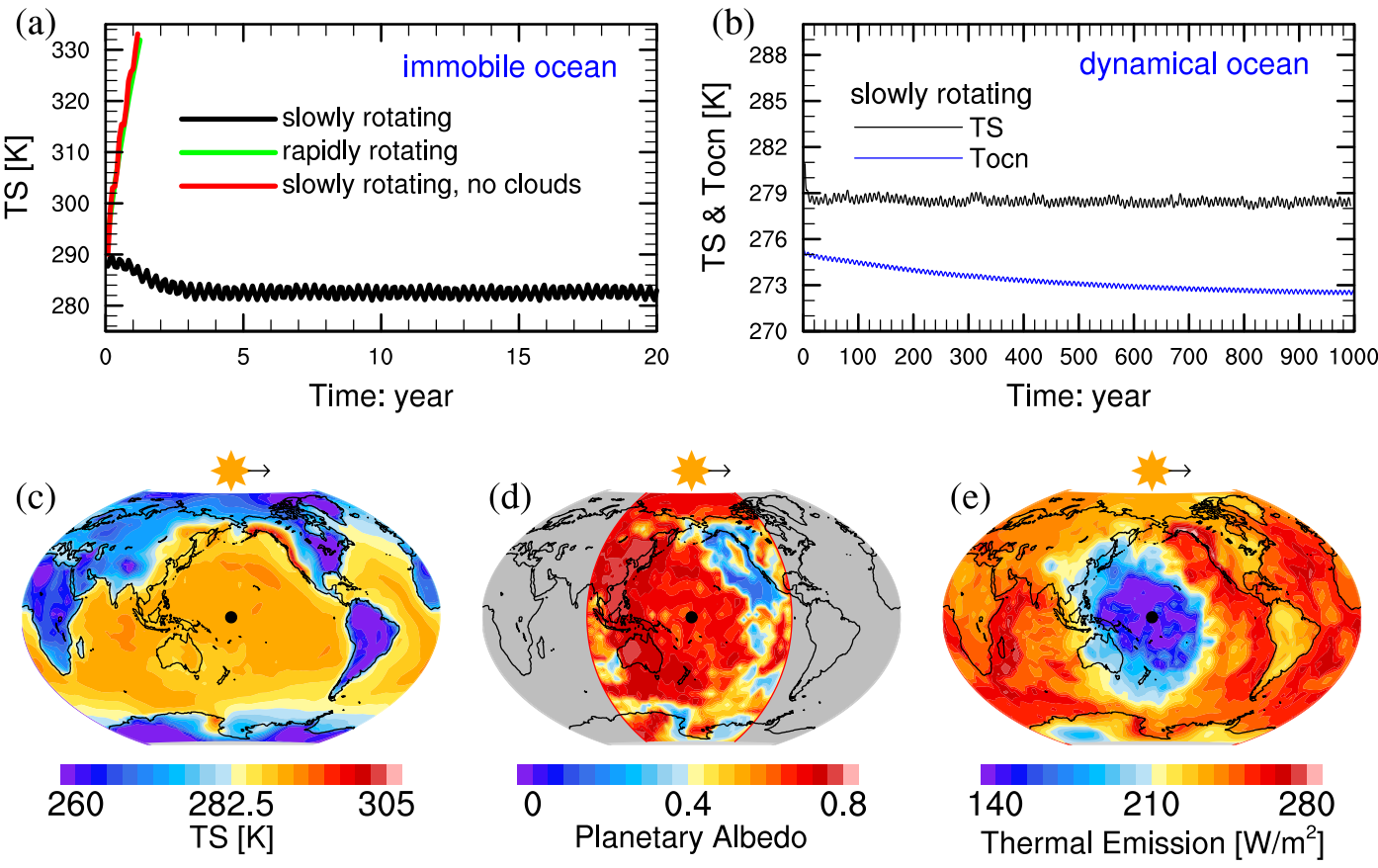

Fig. 4. - The climate of a planet with modern Earth's atmosphere and continental configuration, but in Venus' orbit and with Venus' (slow) rotation rate. (a): Time series of global-mean surface temperature (TS) simulated by the atmospheric model CAM3 for Venus' rotation rate (slowly rotating, black), Earth's rotation rate (rapidly rotating, green), and Venus' rotation rate with clouds artificially set to zero (slowly rotating, no clouds, red). The planet quickly tends toward a runaway greenhouse if it is rapidly rotating or has no clouds, but is habitable if it is slowly rotating. (b): Global-mean TS and vertically-integrated ocean temperature (Tocn) in a coupled ocean-atmosphere simulation using CCSM3 and Venus' rotation rate. (c-e): maps of TS, planetary albedo, and thermal emission to space averaged over 1 day in the coupled CCSM3 simulation. The black dot in (c-e) is the transient substellar point, which moves eastward around the planet with a period of 117 days.

ens, but the zone of ascent spreads out, which leads to a broader area of high relative humidity (Fig. 2h) and high cloud coverage (Fig. 2d). Additionally, the cloud water content increases, making individual clouds optically thicker. As a result, the planetary albedo increases with $S_{0}$ (Fig. 1d).

Our simulations indicate that the inner edge of the habitable zone is strongly dependent on planetary rotation rate. Numerous discovered exoplanets that were previously considered uninhabitable may be within the habitable zon $\$ 3$ if they rotate slowly (Fig. 3). Our simulations yield an inner edge of the habitable zone for rapidly rotating planets approximated by the curve

$$
\begin{aligned}
& S_{\text {rap }}=1.2138+ 9.8344 \times 10^{-5}\left(T_{\text {eff }}-5780\right)+ \\
& 8.8000 \times 10^{-9}\left(T_{\text {eff }}-5780\right)^{2},
\end{aligned}
$$

and for slowly rotating planets

$$
\begin{aligned}
S_{\text {slow }}=2.2296+ & 2.8056 \times 10^{-4}\left(T_{\text {eff }}-5780\right)+ \\
& 1.1308 \times 10^{-8}\left(T_{\text {eff }}-5780\right)^{2},
\end{aligned}
$$

where $S_{\text {rap }}$ and $S_{\text {slow }}$ are stellar fluxes divided by $1360 \mathrm{~W} \mathrm{~m}^{-2}$ and $T_{\text {eff }}$ is the temperature of the star. We note that our $S_{\text {rap }}$ is slightly larger than that of Wolf \& Toon (2014), who also use CAM3, but have implemented a more sophisticated radiative transfer scheme, and use different surface boundary conditions, orbital parameters, and trace gas concentrations (Fig. 3).

3 Our simulations generally have low stratospheric water vapor (Table 1), but a full investigation of water loss on slowly rotating planets is beyond the scope of this paper.
Although the values of $S_{\text {rap }}$ and $S_{\text {slow }}$ estimated using different GCMs and assumptions will likely differ by $\approx 10 \%$, the robust cloud processes we describe should always make $S_{\text {slow }}$ significantly larger than $S_{\text {rap }}$.

Our results suggest that a planet at Venus' distance from a Sun-like star could be habitable if it rotated slowly and had an Earth-like atmosphert. We checked this using CCSM3 to simulate the climate a planet like Earth would have with Venus' present orbital parameters and rotation rate $\left(P_{\text {rot }}=-243\right.$ days). Although the stellar flux is 1.92 times modern Earth's, the planetary albedo is 0.65 so that the planet absorbs slightly less radiation than modern Earth and the maximum TS is only $306 \mathrm{~K}$ (Fig. 4). The slow rotation and consequent stabilizing cloud feedback are the keys to preventing the planet from entering a runaway greenhouse at this high stellar flux. If either $P_{\text {rot }}$ is decreased to 1 day or the clouds are switched off, the model tends toward a runaway greenhouse (Fig. 4a). Further simulations show that the planet tends toward a runaway greenhouse if either the day length or the Coriolis parameter are changed to be Earth-like (and the other is held constant). We have performed a large variety of sensitivity tests that demonstrate the robustness of our conclusion that an Earth-like planet in Venus' orbit would likely be habitable (Table 1). These ideas could eventually be tested by using the James Webb Space Telescope to look for weak thermal emission at the substellar point (Fig. 4e) of detected exoplanets (Yang et al. 2013).

Our work has important implications for the evolu-

4 We note that Leconte et al. (2013b) speculate that rotation rate could be important for the history of habitability on Venus. 
Table 1: Simulated climates of a planet with Venus' orbit and (slow) rotation rate and with an Earth-like atmosphere. By default, $\mathrm{S}_{0}=2615 \mathrm{~W} \mathrm{~m}^{-2}, P_{\text {rot }}=-243$ days, and $P_{\text {orb }}=225$ days.

\begin{tabular}{|c|c|c|c|c|c|c|}
\hline Group & Model & Experimental Design & $\begin{array}{l}\alpha_{p}{ }^{a} \\
0-1\end{array}$ & $\begin{array}{l}\mathrm{G}^{b} \\
\mathrm{~K}\end{array}$ & $\begin{array}{c}\mathrm{V}\left(\mathrm{H}_{2} 0\right)^{c} \\
\text { ppmv }\end{array}$ & $\begin{array}{r}\mathrm{TS}^{d} \\
\mathrm{~K}\end{array}$ \\
\hline $1^{e}$ & CAM3 & default (Earth's continental configuration) & 0.64 & 30 & 1.8 & 282 \\
\hline 1 & CAM3 & no continents (aqua-planet) & 0.58 & 39 & 6.5 & 303 \\
\hline 1 & CAM4 & no continents (aqua-planet) & 0.59 & 38 & 49 & 300 \\
\hline 1 & CCSM3 & dynamical ocean & 0.64 & 24 & 152 & 278 \\
\hline 1 & CCSM3 & dynamical ocean $(4 \mathrm{~km})$, no continents & 0.64 & 29 & 0.4 & 283 \\
\hline $2^{f}$ & CAM3 & default (Earth's continental configuration) & 0.65 & 31 & 2.0 & 284 \\
\hline 2 & CAM3 & switch off sea ice module & 0.65 & 31 & 2.0 & 284 \\
\hline 2 & CAM3 & no continents (aqua-planet) & 0.59 & 41 & 7.5 & 303 \\
\hline 2 & CAM3 & no continents, increase the model resolution & 0.62 & 46 & 137 & 307 \\
\hline 2 & CAM3 & decrease the model time step & 0.64 & 32 & 2.1 & 284 \\
\hline 2 & CAM3 & cloud ice particle size $\times 0.5$ & 0.66 & 32 & 10 & 282 \\
\hline 2 & CAM3 & cloud ice particle size $\times 2.0$ & 0.63 & 31 & 0.6 & 285 \\
\hline 2 & CAM3 & cloud liquid particle size $\times 0.5$ & 0.67 & 29 & 0.5 & 276 \\
\hline 2 & CAM3 & cloud liquid particle size $\times 2.0$ & 0.58 & 43 & 454 & 306 \\
\hline 2 & CAM3 & decrease the mixed layer depth to $1 \mathrm{~m}$ & 0.64 & 26 & 4.1 & 280 \\
\hline 2 & CAM3 & increase the mixed layer depth to $200 \mathrm{~m}$ & 0.65 & 31 & 1.6 & 283 \\
\hline 2 & CAM3 & increase the mixed layer depth to $500 \mathrm{~m}$ & 0.65 & 32 & 1.7 & 284 \\
\hline 2 & CAM3 & increase the $\mathrm{CO}_{2}$ concentration to 0.1 bar & 0.63 & 56 & 158 & 310 \\
\hline 2 & CAM3 & increase the surface pressure to 2 bars & 0.65 & 36 & 0.3 & 287 \\
\hline 2 & CAM3 & increase the surface pressure to 5 bars & 0.65 & 49 & 0.3 & 301 \\
\hline 2 & CAM3 & increase gravity to $1.5 \mathrm{~g}_{\oplus}$ & 0.64 & 29 & 0.7 & 283 \\
\hline 2 & CAM3 & increase the radius to $2.0 R_{\oplus}$ & 0.65 & 31 & 2.0 & 284 \\
\hline 2 & CAM3 & increase the obliquity from $0^{\circ}$ to $23.5^{\circ}$ & 0.65 & 31 & 1.9 & 283 \\
\hline 2 & CAM3 & increase the eccentricity from 0 to 0.2 & 0.61 & 41 & 611 & 301 \\
\hline $3^{g}$ & CAM3 & $\mathrm{S}_{0}=1900 \mathrm{~W} \mathrm{~m}^{-2}, P_{\text {rot }}=8$ days & \multicolumn{4}{|c|}{ tends to runaway warming } \\
\hline 3 & CAM3 & $\mathrm{S}_{0}=1900 \mathrm{~W} \mathrm{~m}^{-2}, P_{\text {rot }}=16$ days & 0.46 & 41 & 0.6 & 301 \\
\hline 3 & CAM3 & $\mathrm{S}_{0}=2615 \mathrm{~W} \mathrm{~m}^{-2}, P_{\text {rot }}=32$ days & \multicolumn{4}{|c|}{ tends to runaway warming } \\
\hline 3 & CAM3 & $\mathrm{S}_{0}=2615 \mathrm{~W} \mathrm{~m}^{-2}, P_{\text {rot }}=48$ days & 0.63 & 32 & 2.4 & 287 \\
\hline
\end{tabular}

a. $\alpha_{p}$ : planetary albedo.

b. G: global-mean greenhouse effect.

c. $\mathrm{V}\left(\mathrm{H}_{2} \mathrm{O}\right)$ : stratospheric vapor content in units of parts per million by volume (ppmv).

d. TS: global-mean surface temperature.

e. Group 1: $\mathrm{pCO}_{2}=400$ ppmv, $\mathrm{pCH}_{4}=1 \mathrm{ppmv}, \mathrm{pN}_{2} \mathrm{O}=0$.

f. Group 2: $\mathrm{pCO}_{2}=500 \mathrm{ppmv}, \mathrm{pCH}_{4}=10 \mathrm{ppmv}, \mathrm{pN}_{2} \mathrm{O}=1 \mathrm{ppmv}$.

g. Group 3: same as default sets of Group 1, except varying $\mathrm{S}_{0}$ and/or $P_{\text {rot }}$.

tion of Venus. Deuterium enrichment in Venus' atmosphere suggests that it may have started with an ocean and gone through a runaway greenhouse Donahue et al. 1982). Our results suggest that if the runaway happened near the beginning of the solar system, Venus would have had to have a rotation period less than a few weeks, and if the runaway occurred recently, Venus would have had to have a rotation period less than a few months (Fig. 1, Table 1). Any water would then be photodissociated and lost to space, and large amounts of $\mathrm{CO}_{2}$ would accumulate in the atmosphere since silicate weathering would not occur if there were no surface water (Kasting 2010). Tidal interactions could later slow Venus' rotation rate to its present value (Correia \& Laskar 2001), but it would be too late for Venus to return to habitable conditions. If instead all of the water on Venus were lost through hydrodynamic escape during formation and Venus did not start with oceans (Hamano et al. 2013), then the rotation rate would not need to have changed over its history.

Consideration of Venus shows that slowly rotating planets which our calculations suggest could be habitable will not actually be habitable in all cases. This will depend on whether they started with a rapid rotation rate, if so how long it took for their rotation rate to slow, the rate of water loss if they entered a runaway greenhouse at some point in their history, their (possible) migration history, and the timing and amount of volatile delivery, among other things. This is no different from any previous estimate of habitable zone, since it has always been understood that habitability depends on planetary history in addition to location.

\section{CONCLUSIONS}

This work demonstrates that the inner edge of the habitable zone for slowly rotating planets could be at twice the stellar flux as for rapidly rotating planets. Numerical simulations suggest that the rotation periods of planets at formation could vary between 10 hours and 400 days (Miguel \& Brunini 2010), and tidal interactions can further slow planetary rotation (Lago \& Cazenave 1979). It is therefore probable that a large number of planets rotate slowly enough to have a greatly expanded habitable zone. Additionally, our simulations suggesting that an Earth-like planet with Venus' present orbit and rotation rate would be habitable demonstrate that empirical limits on the habitable zone based on solar system planets (e.g., Kasting et al. 2014) may not be as strong constraints as previously believed, depending on factors such as rotation rate and planetary history. Finally, we note that although we expect our results to be qualitatively robust, the details will differ with other models that have different cloud and radiation schemes. We can hope to resolve this issue by comparing GCMs, applying cloud resolving models to the problem (e.g., Abbot 2014), and 
eventually observing planets using methods such as those suggested by Yang et al. (2013).

Acknowledgments: We are grateful to D. Koll, Y. Wang, Y. Liu, F. Ding, C. Zhou, and C. Bitz for technical assistance and/or helpful discussions. D.S.A. acknowledges support from an Alfred P. Sloan Research Fellowship. This work was completed in part with resources provided by the University of Chicago Research Computing Center.

\section{REFERENCES}

Abbot, D. S. 2014, J. Climate, in press

Abbot, D. S., \& Tziperman, E. 2008, QJRMS, 134, 165

Abe, Y., Abe-Ouchi, A., Sleep, N. H., \& Zahnle, K. J., 2011, Astrobiology, 11, 443

Collins, W. D., Basch, P. J., Boville, B. A., et al. 2004, Technical Note, Document NCAR-TN-464+STR (Boulder, USA: NCAR)

Collins, W. D., Bitz, C. M., Blackmon, M. L., et al. 2006, J. Climate, 19, 2122

Correia, A. C. M., \& Laskar, J. 2001, Nature, 411, 767

Donahue, T. M., Hoffman, J. H., Hodges, R. R., \& Watson, A. J. 1982, Science, 216, 630

Donohoe, A., \& Battisti, D. S. 2011, J. Climate, 24, 4402

Ferreira, D., Marshall, J., O'Gorman, P. A., \& Seager, S. 2014, Icarus, submitted

Hamano, K., Abe, Y., \& Genda, H. 2013, Nature, 497, 607

Harrison, E. F., Minnis, P., Barkstrom, B. R., Ramanathan, V., Cess, R. D., \& Gibson, G. G. 1990, J. Geophys. Res, 95, 18,687

Held, I. M., \& Hou, A. Y. 1980, J. Atmos. Sci., 37, 515

Joshi, M. M., Haberle, R. M., \& Reynolds, R. T. 1997, Icarus, 129,450

Kasting, J. F., Whitmire, D. P., \& Reynolds, R. T. 1993, Icarus, 101, 108

Kasting, J. F. 2010, How to find a habitable planet (Princeton, USA: Princeton University Press)

Kasting, J. F., Kopparapu, R., Ramirez, R. M., \& Harman, C. E. 2014, PNAS, in press

Kopparapu, R. K. 2013, ApJL, 767 L8

Kopparapu, R. K., Ramirez, R., Kasting, J. F., et al. 2013, ApJ, 767,131
Lago B., \& Cazenave A. 1979, Moon and Planets, 21, 127

Leconte, J., Forget, F., Charnay, B., et al. 2013a, A\&A, 554, A69

Leconte, J., Forget, F., Charnay, B., Wordsworth, R., \& Pottier, A. 2013b, Nature, 504, 268

Miguel, Y., \& Brunini, A., 2010, MNRAS, 406, 1935

Nakajima, S., Hayashi, Y.-Y., \& Abe, Y. 1992, J. Atmos. Sci., 49, 2256

Neale, R. B., Richter, J. H., Conley, A. J., et al. 2010, Technical Note, Document NCAR-TN-485+STR (Boulder, USA: NCAR)

Pierrehumbert, R. T. 2010, Principles of Planetary Climate (Cambridge, UK: Cambridge University Press)

Schneider, T. 2006, Annu. Rev. Earth Planet. Sci., 34, 655

Selsis, F., Kasting, J. F., Levrard, B., et al. 2007, A\&A, 476, 1373

Showman, A. P., Wordsworth, R. D., Merlis, T. M., \& Kaspi, Y 2013, in Comparative Climatology of Terrestrial Planets, eds. Mackwell, S. J., et al., University of Arizona Press, Arizona, USA, pp. 277-326.

Williams, D. M., \& Pollard, D. 2002, International Journal of Astrobiology, 1, 61

Williams, D. M., \& Pollard, D. 2003, International Journal of Astrobiology, 2, 1

Wolf, E. T., \& Toon, O. B. 2014, Geophys. Res. Lett., 41, 167

Yang, J., Cowan, N. B., \& Abbot, D. S. 2013, ApJL, 771, L45 Yang, J., \& Abbot, D. S. 2014, ApJ, 784, 155 